\title{
Epitaxial growth with pulsed deposition: Submonolayer scaling and Villain instability
}

\author{
Hinnemann, Berit; Hinrichsen, H.; Wolf, D.E.
}

Published in:

Physical Review E. Statistical, Nonlinear, and Soft Matter Physics

Link to article, DOI:

10.1103/PhysRevE.67.011602

Publication date:

2003

Document Version

Publisher's PDF, also known as Version of record

Link back to DTU Orbit

Citation (APA):

Hinnemann, B., Hinrichsen, H., \& Wolf, D. E. (2003). Epitaxial growth with pulsed deposition: Submonolayer scaling and Villain instability. Physical Review E. Statistical, Nonlinear, and Soft Matter Physics, 67(1), 011602. https://doi.org/10.1103/PhysRevE.67.011602

\section{General rights}

Copyright and moral rights for the publications made accessible in the public portal are retained by the authors and/or other copyright owners and it is a condition of accessing publications that users recognise and abide by the legal requirements associated with these rights.

- Users may download and print one copy of any publication from the public portal for the purpose of private study or research.

- You may not further distribute the material or use it for any profit-making activity or commercial gain

- You may freely distribute the URL identifying the publication in the public portal 


\title{
Epitaxial growth with pulsed deposition: Submonolayer scaling and Villain instability
}

\author{
Berit Hinnemann, ${ }^{1,2}$ Haye Hinrichsen, ${ }^{3}$ and Dietrich E. Wolf ${ }^{1}$ \\ ${ }^{1}$ Institut für Physik, Gerhard-Mercator-Universität Duisburg, 47048 Duisburg, Germany \\ ${ }^{2}$ CAMP, Department of Physics, Technical University of Denmark, DK-2800 Lyngby, Denmark \\ ${ }^{3}$ Theoretische Physik, Fachbereich 8, Universität GH Wuppertal, 42097 Wuppertal, Germany
}

(Received 22 April 2002; published 14 January 2003)

\begin{abstract}
It has been observed experimentally that under certain conditions, pulsed laser deposition (PLD) produces smoother surfaces than ordinary molecular beam epitaxy (MBE). So far, the mechanism leading to the improved quality of surfaces in PLD is not yet fully understood. In the present work, we investigate the physical properties of a simple model for PLD, in which the transient mobility of adatoms and diffusion along edges is neglected. Analyzing the crossover from MBE to PLD, the scaling properties of the time-dependent nucleation density as well as the influence of Ehrlich-Schwoebel barriers, we find that there is indeed a range of parameters, where the surface quality in PLD is better than in MBE. However, since the improvement is weak and occurs only in a small range of parameters we conclude that deposition in pulses alone cannot explain the experimentally observed smoothness of PLD-grown surfaces.
\end{abstract}

DOI: 10.1103/PhysRevE.67.011602

\section{INTRODUCTION}

Pulsed laser depostion (PLD) is an increasingly used growth method [1], which plays an important role in various applications such as the growth of ultrahard carbon films [2], artificially strained superlattices [3], superconducting films, [4], as well as the fabrication of multilayered structures with a chemically complex behavior [5]. In the PLD, the target material is ablated by a pulsed laser and then deposited in pulses on a substrate so that many atoms arrive at the surface simultaneously. Experimentally, each pulse lasts for about a few nanoseconds, and the time between two pulses is of the order of seconds.

PLD differs significantly from ordinary molecular beam epitaxy (MBE), where atoms are deposited continuously. On one hand, the physical conditions for PLD are far less well defined than for MBE. The particles deposited may be atoms, clusters, or even droplets with energies ranging from $0.1 \mathrm{eV}$ to $1000 \mathrm{eV}$. On the other hand, very energetic particles may lead to implantation or surface sputtering effects. Moreover, their kinetic energy is converted into heat at the surface, changing locally the effective mobility of the particles for a short time.

Growth experiments at moderate energies show that the surfaces in PLD and MBE have different morphologies. Recently, Jenniches et al. compared the quality of layer-bylayer growth of $\mathrm{Fe} / \mathrm{Cu}(111)$ at room temperature, using ordinary MBE as well as PLD at energies between 1 and $10 \mathrm{eV}$, where sputtering and implantation effects can be excluded [6]. They observed that in some cases PLD, leads to smoother surfaces than MBE. However, so far the mechanism leading to smoother surfaces in PLD is not yet entirely understood so it is impossible to predict the growth quality for various materials and growth parameters. Therefore, the aim of the present work is to improve our understanding of PLD by investigating the growth morphology for different growth parameters in a simple model.

Recently, Narhe et al. investigated the island statistics in the coalescence regime for a PLD process, where tin droplets are deposited on a sapphire substrate [7]. It was observed
PACS number(s): 81.15.Fg, 64.60.Ht, 68.55.Ac

that the scaling of the droplet size distribution differs significantly from MBE due to the large fraction of multiple droplet coalescence under pulsed vapor delivery. While these results are valid for high deposition energies, the present work investigates a different physical regime, namely, pulsed deposition at low energies of about $0.1 \mathrm{eV}$, where the transient enhancement of the mobility can be neglected. Our aim is to study the scaling properties and, in particular, the influence of the Ehrlich-Schwoebel barrier.

The model investigated in the present work involves pulsed deposition, surface diffusion of adatoms, and nucleation of islands. In our model, the influence of edge diffusion is neglected so that islands grow in a fractal manner before they coalesce. Moreover, we assume that atoms can neither evaporate nor detach from the edges of islands. The purpose of these restrictions is to keep the model as simple as possible.

The model is defined as a solid-on-solid growth process on a square lattice of $L \times L$ sites with integer heights representing the configuration of the adsorbed layer. The particles are deposited in pulses with an intensity $I$, which is defined as the number of particles per unit area deposited per pulse. The duration of a pulse is assumed to be zero and the transient enhancement of the mobility of freshly deposited adatoms is neglected. The model is controlled by three parameters, namely, the intensity $I$ of the pulses, the diffusion constant $D$, and the average flux density of incoming particles $F$. One of these parameters can be fixed by choosing the time scale so that we can use $I$ and $D / F$ as independent parameters. The dynamic rules are defined as follows. (i) In each pulse, $I L^{2}$ atoms are instantaneously deposited at random positions on the surface. (ii) Between two pulses, a time interval $\Delta t=I / F$ elapses, in which adatoms diffuse on the surface with rate $D$. If Ehrlich-Schwoebel barriers are present, the rate at which particles hop down the edge of an island is reduced. (iii) If two atoms at the same height occupy neighboring sites, they stick together irreversibly, forming the nucleus of a new island or attaching to an already existing island.

The paper is organized as follows. In the following sec- 

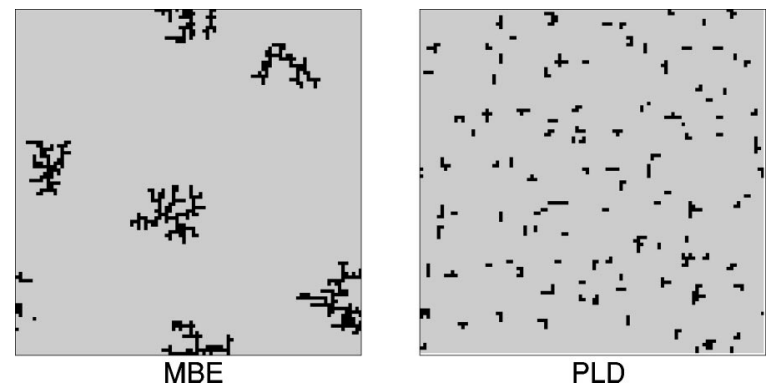

FIG. 1. Molecular beam epitaxy (left) compared to pulsed laser deposition (right) for $D / F=10^{8}$ and $I=0.01$. The figure shows typical configurations after deposition of $0.05 \mathrm{ML}$.

tion, we first recall the properties of PLD without EhrlichSchwoebel barriers and its crossover to MBE in the limit of very low intensities $[8,9]$. In Sec. III, we investigate the scaling behavior of the time-dependent nucleation density in PLD, extending our previous analysis in Ref. [10]. In Sec. IV, we study the influence of Ehrlich-Schwoebel barriers on the morphology of surfaces grown by PLD, finding a parameter range, where layerwise growth is improved compared to $\mathrm{MBE}$. If not stated otherwise, all numerical results are obtained by kinetic Monte Carlo simulations on a lattice of $400 \times 400$ sites. The influence of finite-size effects is discussed in the Appendix.

\section{FROM MBE TO PLD: CROSSOVER AND SCALING}

If the intensity $I$ is very small, PLD behaves essentially in the same way as MBE. In fact, at the lowest possible intensity, where only one atom per pulse is deposited, MBE and PLD are equivalent up to minor statistical differences resulting from finite-size effects (see the Appendix). Using lattices of $400^{2}$ sites, we, therefore, restrict our analysis to the range $D / F \leqslant 10^{7}$, where these finite-size effects are negligible.

At high intensities, the growth morphology of PLD differs significantly from MBE. As shown in Fig. 1, there are much more nucleations at an early stage, although the effective flux of incoming particles is the same in both cases. The two regimes are separated by a crossover at a certain intensity $I_{c}$, where the number of deposited atoms per pulse is of the same order of magnitude as the average adatom density in the corresponding MBE process. Obviously, if the pulse intensity is much higher than the MBE adatom density, the adatoms nucleate much faster forming many small islands. For this reason, PLD is expected to yield more homogeneous surfaces, which explains the technological interest in this method.

Let us now study the crossover from MBE to PLD in more detail $[8,9]$. The adatom density in MBE averaged over space and time is known to scale as $(D / F)^{2 \gamma-1}$ [11], where

$$
\gamma=\frac{1}{d_{f}+4}= \begin{cases}1 / 6 & \text { for compact islands } \\ 0.18 & \text { for DLA fractals [12] }\end{cases}
$$

Thus, the crossover takes place at the critical intensity

$$
I_{c} \propto(D / F)^{2 \gamma-1} .
$$

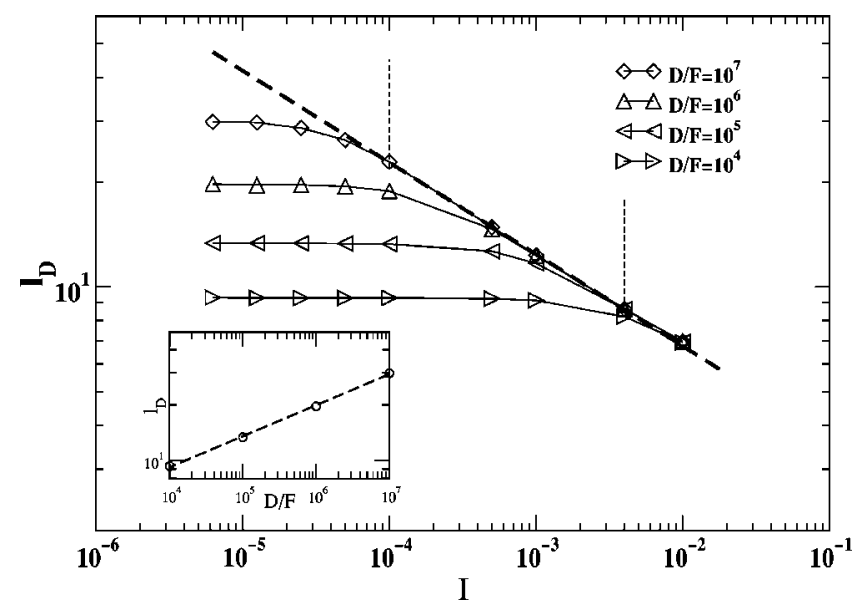

FIG. 2. Average island distance $\ell_{D}$ versus pulse intensity $I$ for different values of $D / F$. The data points between the two dotted vertical lines are used to determine the exponent $\nu$. The dashed line is the corresponding power-law fit with the slope $-\nu=-0.26(1)$. The inset shows the saturation levels of $\ell_{D}$ for small $I$ as a function of $D / F$. The dashed line has slope $\gamma=0.17(1)$.

A quantity which distinguishes the two different growth modes shown in Fig. 1 is the average island distance. Performing numerical simulations, we realized that the scaling regime of this quantity is not only restricted by finite-size effects but also by lattice effects, which become relevant for high intensities as well as for low values of $D / F$, where the average distance of islands is of the order of a few lattice constants. Combining these bounds, we find that for a system with $400^{2}$ sites, finite-size and lattice effects are negligible in the parameter range $10^{4} \leqslant D / F \leqslant 10^{7}$ and $I \leqslant 10^{-2}$.

Figure 2 shows the average island distance as a function of the intensity for various values of $D / F$ at a fixed coverage of 0.2 monolayers (ML). For low intensities $I<I_{c}$, the island distance depends only on $D / F$. Plotting these saturation values versus $D / F$ (shown in the inset of the figure), one recovers the well-known power law for the island distance in $\mathrm{MBE}$,

$$
\ell_{D} \propto(D / F)^{\gamma}
$$

with $\gamma=0.17(1)$. This estimate lies between the values for compact growth and diffusion-limited aggregation [see Eq. (1)], supporting the assumption that the islands are characterized by an effective fractal dimension $1.6<d_{f}<2$.

For intensities $I>I_{c}$, the island distance is independent of $D / F$ and can be described by a power law

$$
\ell_{D} \propto I^{-\nu}
$$

with an exponent $\nu=0.26(1)$. The independence of the island distance of $D / F$ is a result of the high density of adatoms, which nucleate so quickly that they do not make use of their full mobility given by $D / F$. [9]

Combining Eqs. (3) and (4), we obtain the scaling form

$$
\ell_{D} \propto(D / F)^{\gamma} h\left(I / I_{c}\right)
$$




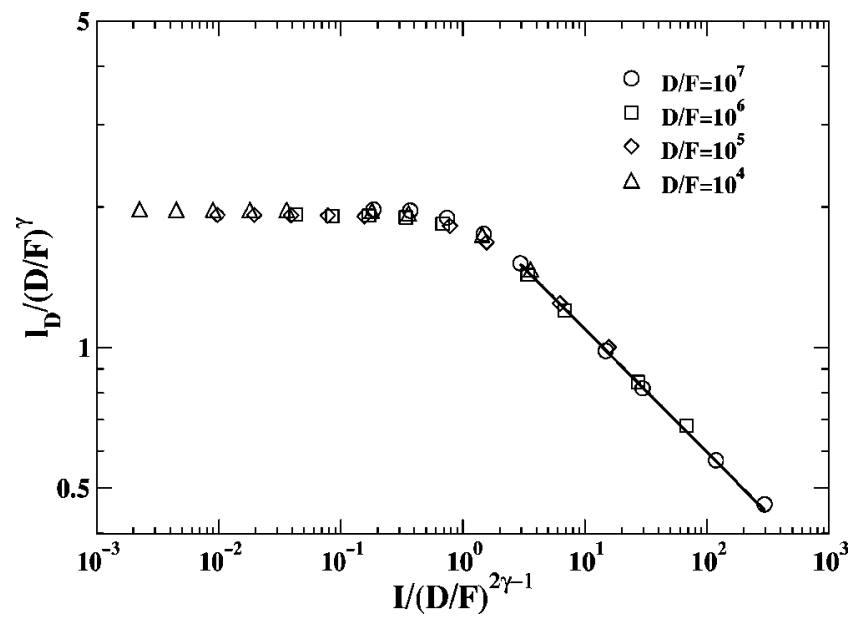

FIG. 3. Data collapse of the curves in Fig. 2 according to Eq. (5) using the exponents $\gamma=0.17$ and $\nu=0.26$. The knee of the curves marks the crossover from MBE- to PLD-like behavior.

where $h$ is a scaling function with the asymptotic behavior

$$
h(y) \sim\left\{\begin{array}{l}
\text { const } \text { for } y \ll 1 \\
y^{-\nu} \text { for } y \gg 1
\end{array}\right.
$$

As shown in Fig. 3, this scaling form leads to a convincing data collapse. Moreover, the independence of $D / F$ at high intensities, together with Eq. (2) implies that

$$
\nu=\frac{\gamma}{1-2 \gamma} \simeq \begin{cases}0.25 & \text { for compact islands } \\ 0.28 & \text { for DLA fractals }\end{cases}
$$

confirming our numerical estimate $\nu=0.26(1)$.

\section{TIME-DEPENDENT NUCLEATION DENSITY}

In a recent paper [10], we investigated the time-dependent nucleation density in PLD, reporting an unusual type of scaling behavior. In order to avoid the crossover to MBE, we considered the limit $D / F \rightarrow \infty$, where $I_{c}=0$. In this limit, all adatoms nucleate or attach to existing islands before the next pulse arrives. Figure 4 shows a log-log plot of the the nucleation density at the bottom layer $n(I, \Theta)$ as a function of the coverage $\Theta=F t$ for various intensities $I$. Obviously, this quantity does not display ordinary power-law scaling since it is impossible to collapse the curves by shifting them horizontally and vertically. However, in Ref. [10], we observed that the normalized nucleation density

$$
M(I, \Theta)=n(I, \Theta) / n(I, 1)
$$

obeys an unusual logarithmic scaling law of the form

$$
\ln M(I, \Theta) \simeq(\ln I) g(\ln \Theta / \ln I) .
$$

This scaling form was also proposed by Kadanoff et al. and Tang in the context of multiscaling in self-organized criticality $[13,14]$. As shown in Fig. 5, this scaling form leads to a convincing data collapse. More recently, the same type of scaling has also been observed in one-dimensional systems,

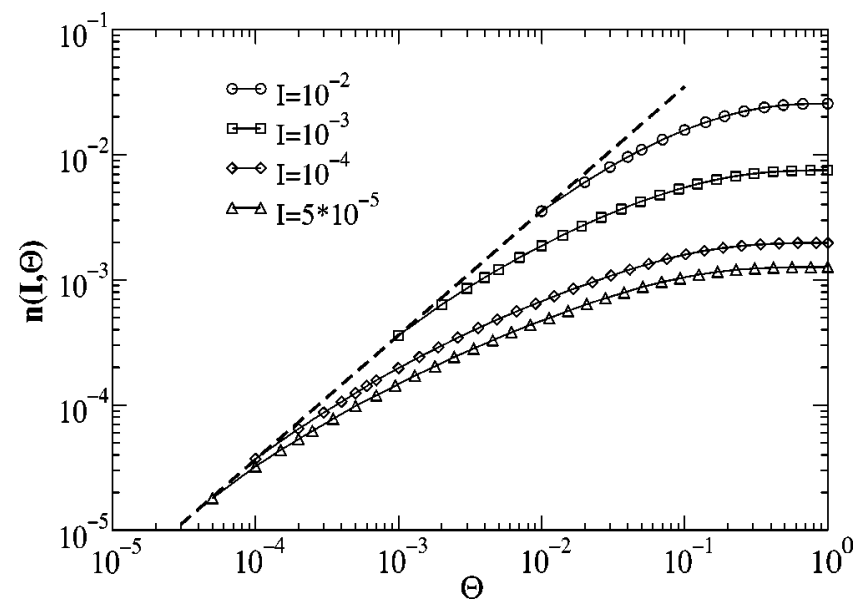

FIG. 4. The nucleation density at the bottom layer versus monolayer time during the deposition of one monolayer. The dashed line has the slope 1 .

so that we can rule out logarithmic corrections at the marginal dimension of random walks as the origin for this type of nonconventional scaling $[15,16]$. In Fig. 5, the left terminal points of the curves at $g(1)=0.44(2)$ can be used to determine $\gamma$ as follows. It is known that the nucleation density after the first pulse grows linearly with the intensity [10], while the nucleation density after completion of one monolayer grows as $n(I, 1) \propto I^{-2 \nu}$ [see Eq. (4)]. Therefore, the normalized nucleation density after the first pulse scales as $M(I, I) \propto I^{1-2 \nu}$ so that

$$
g(1)=1-2 \nu=\frac{1-4 \gamma}{1-2 \gamma} \text {. }
$$

Solving this equation, we obtain $\gamma=0.179(4)$ in agreement with the estimate in Fig. 2.

In order to understand how this unusual scaling behavior for PLD crosses over to the ordinary power-law scaling of

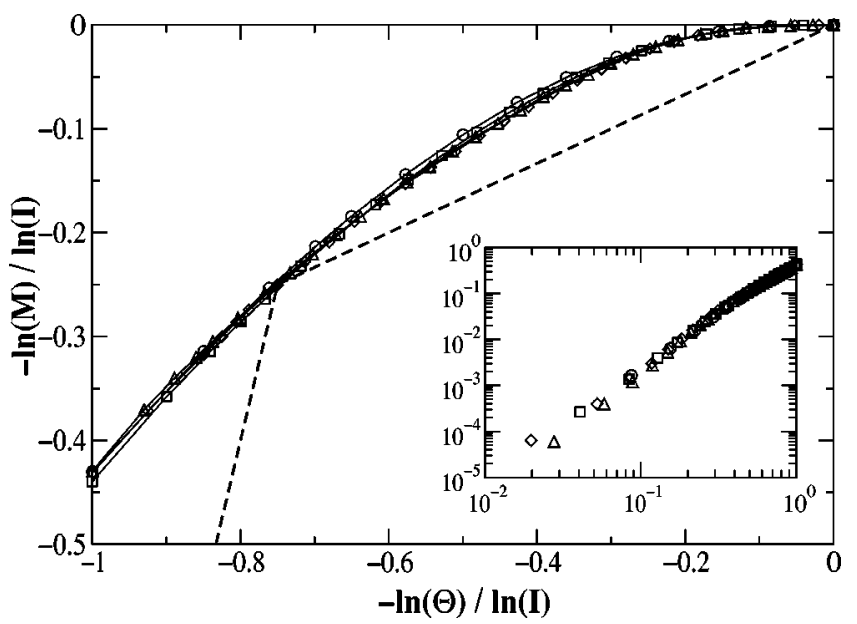

FIG. 5. Data collapse of the curves in Fig. 4 according to the scaling form (9). The figure visualizes the scaling function $g(x)$ $=\ln M / \ln I$ versus $x=\ln \Theta / \ln I$. The inset shows a doublelogarithmic plot of $\ln M / \ln I$ vs $\ln \Theta / \ln I$. The line is slightly curved with local slopes varying from 2.4 to 2.0 . 
MBE, let us now turn to the case of finite $D / F$, where the system is characterized by a typical length scale $\ell_{0}$ $\sim(D / F)^{1 / 4}$. Generalizing the results of Ref. [10], we consider islands with an arbitrary fractal dimension, i.e., we regard $\gamma$ as a free parameter.

In MBE, the nucleation density is known to exhibit ordinary power-law scaling of the form

$$
n\left(\ell_{0}, \Theta\right)=\ell_{0}^{-2} f\left(\Theta \ell_{0}^{2}\right),
$$

where $f$ is a scaling function with the asymptotic behavior [17]

$$
f(z) \propto\left\{\begin{array}{l}
z^{3} \text { for } 0 \leqslant z \ll 1 \\
z^{1 / 3} \text { for } 1 \ll z \lesssim z_{\max }
\end{array}\right.
$$

The upper bound $z_{\max }$ is determined by the condition that the whole surface is covered by islands so that no further nucleation in the respective layer is possible [11]. Because of $I_{c}$ $\sim(D / F)^{2 \gamma-1}$, the length scale $\ell_{0}$ is related to the crossover intensity by

$$
\ell_{0} \sim I_{c}^{-1 /(4-8 \gamma)} .
$$

Using this scaling form, we obtain the expression

$$
M\left(I_{c}, \Theta\right)=\left\{\begin{array}{l}
\Theta^{3} I_{c}^{-(3-1 / 3) /(2-4 \gamma)} \text { for } 0 \leqslant z \ll 1 \\
\Theta^{1 / 3} \text { for } 1 \ll z \lesssim z_{\max },
\end{array}\right.
$$

where $z=\Theta I_{c}^{-1 /(2-4 \gamma)}$. Taking the logarithm and extrapolating to $z=\ell_{0}^{2}$, we arrive at

$$
\frac{\ln M\left(I_{c}, \Theta\right)}{\ln I_{c}}=\left\{\begin{array}{l}
3 \frac{\ln \Theta}{\ln I_{c}}+\frac{4}{6 \gamma-3} \quad \text { for } \frac{1}{2-4 \gamma} \ll \frac{\ln \Theta}{\ln I_{c}} \\
\frac{\ln \Theta}{3 \ln I_{c}} \quad \text { for } 0 \ll \frac{\ln \Theta}{\ln I_{c}} \ll \frac{1}{2-4 \gamma} .
\end{array}\right.
$$

In the limit $I \simeq I_{c} \rightarrow 0$ (i.e., $D / F \rightarrow \infty$ ), the crossover between both regimes becomes sharper and converges to a piecewise linear curve, which is shown in Fig. 5 as a dashed line. The crossover point is located at

$$
\begin{gathered}
\ln \Theta_{c} / \ln I_{c}=1 /(2-4 \gamma), \\
\ln M\left(I_{c}, \Theta\right) / \ln I_{c}=1 /(6-12 \gamma) .
\end{gathered}
$$

Surprisingly, the crossover point lies on the collapsed curves for PLD within numerical errors. This is plausible for the following reasons. On one hand, the PLD curve must be an upper bound for the MBE curve since the island density in PLD is always larger. On the other hand, if the gap between the two extrapolated curves did not close at the crossover between PLD and MBE behavior, it would imply that there is an additional characteristic length in the system, for which we have no evidence.

Furthermore, we note that the scaling function itself roughly follows a power law,

$$
g(z) \simeq g(1) z^{\beta} .
$$

As shown in the double-logarithmic inset of Fig. 5, the effective exponent $\beta$ varies between 2.4 and 2.0. In order to verify this estimate, we derive the exponent $\beta$ from Eq. (16) by assuming that the crossover point lies exactly on the collapsed curves for PLD. This leads to an expression for $\beta$ in terms of the exponent $\gamma$, namely,

$$
\beta=\frac{\ln (6-24 \gamma)}{\ln (2-4 \gamma)} \simeq\left\{\begin{array}{l}
2.4 \text { for compact islands } \\
2.15 \text { for DLA fractals. }
\end{array}\right.
$$

Since this is just the range in which the numerical values for $\beta$ vary, we are led to the conclusion that the effective fractal dimensions of the islands for low and high coverages are different. For low coverages, the islands are spaced relatively far apart so that the growth is DLA-like while for high coverages, the islands coalesce and become more and more compact. Moreover, one has to take into account that for very small islands, the lattice cutoff may influence the effective fractal dimension.

\section{INFLUENCE OF EHRLICH-SCHWOEBEL BARRIERS}

In most experimental situations, interlayer transport is reduced by an additional energy barrier $E_{\mathrm{ES}}$, which the atoms have to overcome when hopping down an edge of an island [18]. This barrier is called Ehrlich-Schwoebel (ES) barrier and is of the order of about $0.1 \mathrm{eV}$ for metals [19]. A useful measure for the influence of this barrier is the Schwoebel length $\ell_{\mathrm{ES}}$, which is defined [20] as

$$
\ell_{\mathrm{ES}}=a \exp \left[\frac{E_{\mathrm{ES}}}{k_{\mathrm{B}} T}\right]
$$

where $a=1$ denotes the lattice constant. For MBE, it is known that Ehrlich-Schwoebel barriers impede interlayer transport. This leads to a growth instability which was predicted and first theoretically investigated by Villain [21] followed by others [22] and has also been observed experimentally [23]. In contrast to MBE, not much is known about the influence of Ehrlich-Schwoebel barriers on PLD. However, it has been observed experimentally that PLD leads to a better growth of results than MBE in certain situations where Ehrlich-Schwoebel barriers are present [6].

The influence of ES barriers on PLD is twofold. On one hand, for high intensities, many adatoms are deposited on the same island, which should increase the influence of ES barriers. On the other hand, in PLD the islands are much smaller so that adatoms tend to leave an island very quickly, thereby reducing the influence of ES barriers. Therefore, the question arises whether the experimentally observed improved quality of layer-by-layer growth in PLD can be related to a reduced influence of ES barriers and whether it is possible to choose the parameters of the model in such a way that PLD produces smoother surfaces than MBE.

This question has been addressed previously by Schinzer et al. [24]. They studied the special case, where one intense pulse $(0.23 \mathrm{ML})$ is deposited at the beginning of each monolayer, while the remaining atoms are deposited with a continuous flux. The aim was to check an idea of Rosenfeld 
et al. [25] that it should improve the smoothness of the surface, if the islands are forced to be much smaller than the diffusion length of the atoms deposited after the pulse. Surprisingly, Schinzer et al. [24] found that this is not the case in their simulation. On the contrary, in the presence of the pulse, the surface became rougher.

A key concept for describing the influence of ES barriers is the time it takes before the first nucleation in the second layer takes place. Layer-by-layer growth requires that nucleations in the second layer do not start significantly before the first layer is completed [21]. In the case of MBE, the study of the second-layer nucleation time turned out to be very useful in order to predict the growth mode for a given set of parameters $[26,27]$. In the following, we demonstrate that this concept can be successfully applied to PLD as well. To this end, we first investigate the growth behavior of PLD in the limit of $D / F \rightarrow \infty$. It will be shown that for any finite EhrlichSchwoebel barrier, MBE produces a better layer-by-layer growth than PLD in agreement with the finding of Schinzer et al. [24]. Then we compare MBE and PLD for different values of $I$ and $D / F$ over a wide range of Ehrlich-Schwoebel barriers. It turns out that there is indeed a regime where PLD produces better growth results than MBE.

\section{A. Infinite $D / F$}

We start by explaining why in the limit $D / F \rightarrow \infty$, PLD does not delay the Villain instability in spite of the island size reduction compared to MBE, provided that the ES barrier is sufficiently high and not leaky, e.g., at kink sites. In this limit, the time scales are separated as follows. Because of the high ES barrier the nucleation time of adatoms on top of islands is much smaller than their residence time, while for $D / F \rightarrow \infty$, the residence time is in turn much smaller than the time interval between two pulses. This means that we can restrict our analysis to a single pulse.

The second layer nucleation probability $p_{\text {nuc }}(I, A)$ is defined as the probability that there is at least one nucleation event on islands with area $A$ after deposition of a pulse with intensity $I$. For high ES barrier, this means that during a single pulse at least two atoms have to be deposited on the same island. Obviously, the probability of depositing $k$ atoms on an island with area $A$ during one pulse is given by a Poisson distribution with average $I A$,

$$
p_{k}=\frac{(I A)^{k}}{k !} e^{-I A} \text {. }
$$

Hence, the nucleation probability can be expressed as

$p_{\text {nuc }}(I, A)=1-p_{0}-p_{1}=1-e^{-I A}(1+I A) \simeq I^{2} A^{2}+O\left(I^{3} A^{3}\right)$.

Figure 6(a) shows the numerical results for $p_{\text {nuc }}$ for $I=0.01$ and different ES barriers. As can be seen, for increasing ES barrier, the measured curves approach the predicted one, Eq. (21), which is shown as a dashed line. The agreement is good for $\ell_{E S} \geqslant 10^{3}$, where the nucleation time is much shorter than the residence time. However, the agreement is not convincing for island areas $A<10$, where the discrete lattice spacing
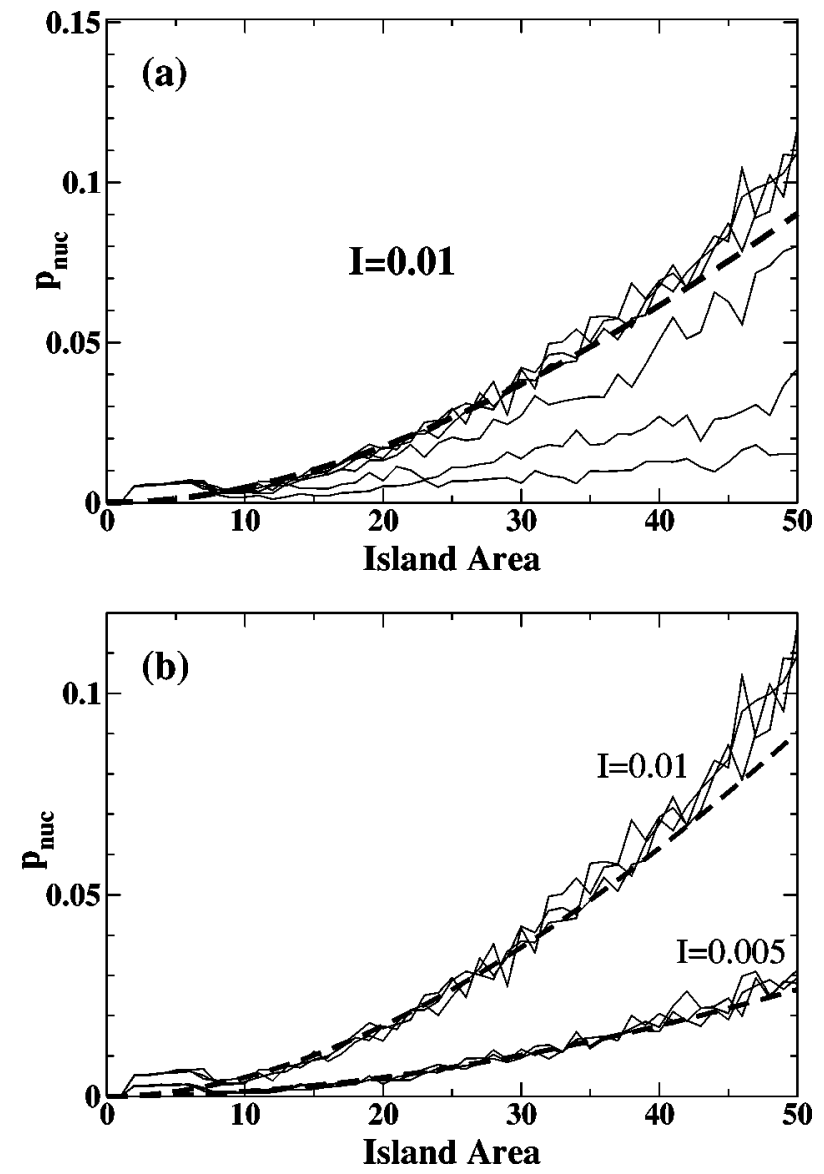

FIG. 6. Numerical measurement of the second-layer nucleation probability compared to the theoretical prediction. After each pulse $p_{\text {nuc }}$ is estimated as the fraction of islands with area $A$, where a second-layer nucleation happened for the first time, averaging over several runs. The island area is restricted to $A \leqslant 50$, where coalescence does not yet play a role. (a) The second layer nucleation probability $p_{\text {nuc }}$ for $I=0.01$ versus island size $A$ for different Ehrlich-Schwoebel barriers in the limit $D / F \rightarrow \infty$. From bottom to top the Schwoebel lengths are $\ell_{E S}=10^{0}, 10^{1}, 10^{2}, 10^{3}, 10^{4}, 10^{5}$. (b) Plot of $p_{\text {nuc }}$ versus $A$ for two different intensities and for high Ehrlich-Schwoebel barriers $\ell_{\mathrm{ES}}=10^{3}, 10^{4}, 10^{5}$.

starts to play a role. Moreover, the upper curves deviate for $A>40$ due to coalescence of large islands. This explanation is supported by the results shown in Fig. 6(b), where two different intensities are compared. Indeed, for the lower intensity $I=0.005$, where coalescence starts at larger island sizes, the measured and the predicted curves agree much better. Thus, we conclude that the second layer nucleation probability $p_{\text {nuc }}$ is adequately described by Eq. (21).

With Eq. (21), we can now answer that which of the opposing trends in PLD-island size reduction and increasing nucleation probability on top of islands-will dominate. In the limit $D / F \rightarrow \infty$, where the critical intensity $I_{c}$ tends to zero, the island area scales as $A \sim \ell_{D}^{2} \sim I^{-2 v}$ for all intensities. Together with Eq. (21) one obtains to leading order

$$
p_{\mathrm{nuc}} \propto I^{2(1-2 \nu)} .
$$

Using the previous estimate $\nu \simeq 0.26$, the exponent is given 

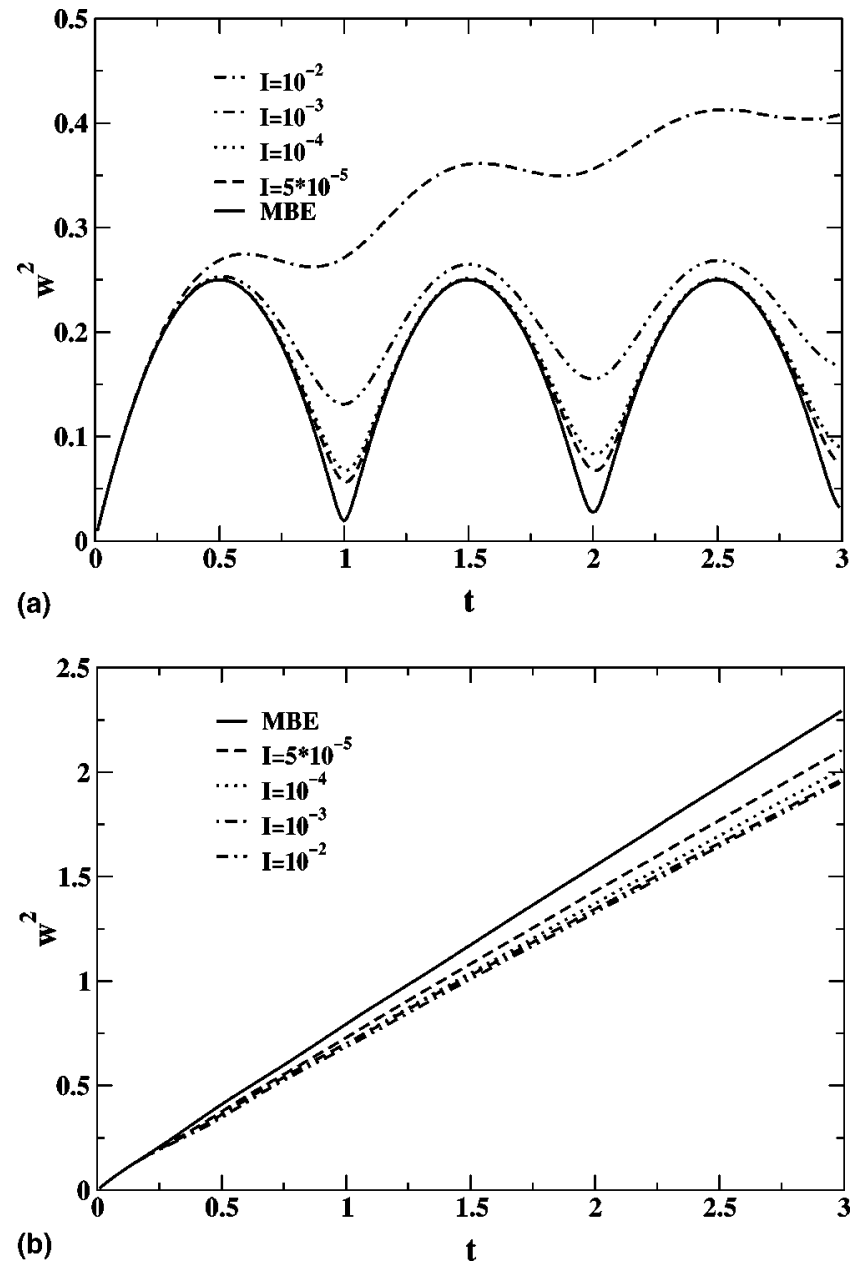

FIG. 7. (a) Squared surface width versus time for MBE and PLD for different intensities. The growth parameters are $D / F$ $=10^{8}$ and $\ell_{\mathrm{ES}}=1$. (b) Squared surface width versus intensity for $D / F=10^{8}$ and $\ell_{\mathrm{ES}}=10^{4}$.

by $2(1-2 \nu) \simeq 1$. Therefore, the second layer nucleation probability $p_{\text {nuc }}$ grows with increasing intensity, enhancing the Villain instability. Contrarily, MBE with an infinite $D / F$ always shows perfect layer-by-layer growth, even for high but finite ES barriers. Thus, we conclude that in the limit $D / F \rightarrow \infty$, PLD cannot improve layer-by-layer growth. In order to apply this result to practical situations, one has to find the lower boundary for $D / F$, below which the behavior is different. This question will be addressed now.

\section{B. Comparison of PLD and MBE for finite $D / F$}

For perfect layer-by-layer growth, the squared surface width $w^{2}=\left(\langle h\rangle^{2}-\left\langle h^{2}\right\rangle\right)$ is known to oscillate between zero for completed monolayers and 1/4 for half monolayers [28]. Without ES barriers, such oscillations can be seen in MBE as well as in PLD. As shown in Fig. 7(a), they are most pronounced in MBE while in PLD, they become more and more damped as the intensity increases. Moreover, it can be seen that in PLD without ES barriers, the roughness always increases with increasing pulse intensity.

Figure 7(b) shows the corresponding result for a very high Ehrlich-Schwoebel length $\ell_{\mathrm{ES}}=10^{4}$. There are no oscilla-
$I=0.1$

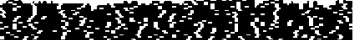

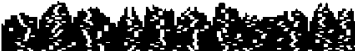

$I=0.01$
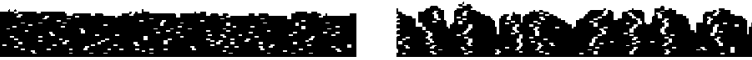

$I=0.001$

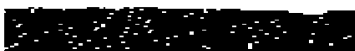

$l_{\mathrm{Es}}=1$

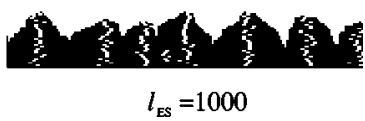

FIG. 8. 1+1-dimensional PLD after deposition of $20 \mathrm{ML}$ for different pulse intensities. The nucleation sites are shown as white dots. Left: without Ehrlich-Schwoebel barrier the roughness increases with increasing intensity. Right: if the Ehrlich-Schwoebel barrier is high enough, the autocorrelation among nucleation sites is more pronounced than for $\ell_{\mathrm{ES}}=1$, and the roughness decreases with increasing intensity.

tions since the surface roughens very quickly due to the Villain instability [21]. Surprisingly, the width is now maximal for $\mathrm{MBE}$ and decreases with increasing pulse intensity, i.e., the trend is reversed. This reversal can even be observed visually by monitoring the interface at different intensities, as shown in Fig. 8.

Let us now investigate this reversal in more detail. Since the oscillations in PLD are extremely weak or not present at all, a numerical measurement of the damping time (such as in Ref. [29] for MBE) is not feasible. Instead, we monitored the squared surface width after deposition of a two monolayers - a coverage which is also relevant for experimental applications [6]. Although the choice of two monolayers is arbitrary and does not permit a rigorous quantitative analysis, this criterion is very simple and tells us, for which parameters PLD produces smoother surfaces than MBE.

The roughness after deposition of two monolayers is plotted in Fig. 9. In agreement with previous results for MBE [30], all curves increase monotonously, i.e., the roughness increases with increasing Ehrlich-Schwoebel barrier. However, the curves for MBE and PLD cross each other. The typical ES barrier, where this crossing takes place, varies roughly as $\ell_{\mathrm{ES}} \approx(D / F)^{1 / 2}$. This observation is in agreement

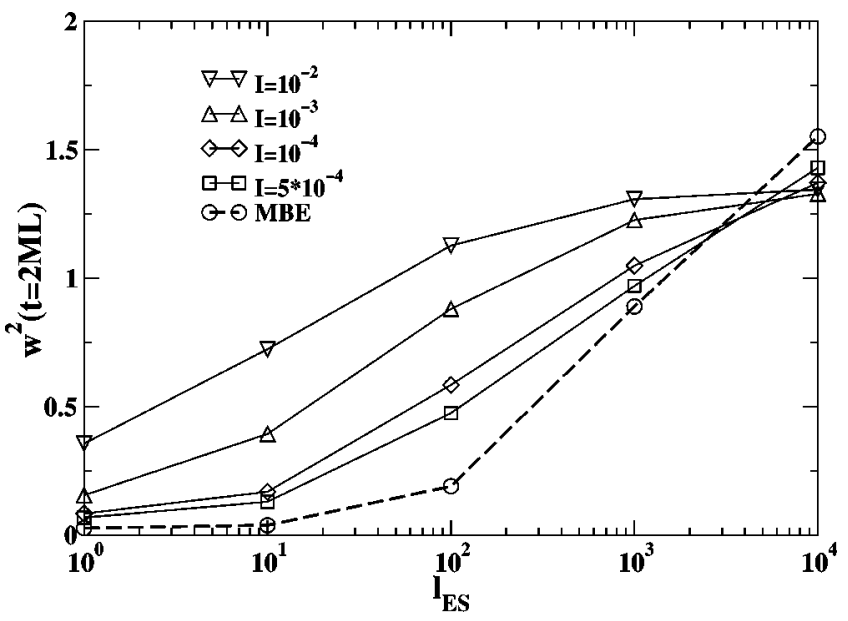

FIG. 9. The squared surface width at $\Theta=2 \mathrm{ML}$ versus the Schwoebel length for MBE and PLD with various intensities and for $D / F=10^{8}$. The curve for MBE is dashed. 
with our previous result that for $D / F \rightarrow \infty$, surfaces grown by MBE are always smoother compared to PLD.

Thus, there is indeed a range of parameters where PLD with a high intensity produces smoother surfaces than MBE, even if the atoms are deposited with thermal energy. However, the Ehrlich-Schwoebel barriers are unphysically large (typical experimental values are $\ell_{E S} \approx 1, \ldots, 10$ ). Therefore we believe that nonthermal energy deposition effects are important for explaining the experimentally observed growth improvement in PLD.

Nevertheless, it is interesting to address the question, what causes the crossing of the curves in Fig. 9. As we have explained, second layer nucleation becomes more likely the larger the pulse intensity is in spite of the decreasing island size. Therefore, one would always expect the roughness to increase with pulse intensity, as it is indeed the case for sufficiently small Ehrlich-Schwoebel barriers. For $\ell_{\mathrm{ES}} \rightarrow \infty$, however, there is no interlayer transport and the roughness is the same as in random deposition, irrespective of the pulse intensity. Thus, the curves in Fig. 9 are expected to saturate eventually at the same roughness. Thus, the observed reversal results from an interplay of a large but finite ES barrier and a finite value of $D / F$.

To understand the reversal, let us consider how the Villain instability unfolds for large but finite ES barriers. Depending on the intensity, the first pulse leads to the formation of many small islands. After the first pulse, there are two temporal regimes of different roughening behavior. Because of the Villain instability, effective uphill currents lead to a quick formation of large mounds. As new nucleations preferentially take place on top of these mounds, the nucleations are vertically aligned [31], as shown on the right-hand side of Fig. 4(b). Therefore, the number and the lateral size of the mounds is essentially determined by the initial configuration of small islands after the first pulse. This determines at least for transient times also the height of the mounds and, therefore, the roughness of the surface. In our simulation model, we do not observe slope selection. In a more realistic case, one would expect that the mounds grow until their edges reach a critical slope, where the uphill current becomes zero. Then the process enters a different temporal regime where the mounds compete with one another, leading to an effective coarsening process where the roughness increases only slowly.

By increasing the pulse intensity, second layer nucleations become more likely so that the Villain instability is accelerated. However, the typical roughness, from where on the process enters the second temporal regime of slow coarsening depends mainly on the initial density of islands after the first pulse. Therefore, this typical roughness decreases with increasing intensity. After sufficiently long time, this effect dominates and leads to the observed crossings of the curves in Fig. 9. Obviously, this mechanism works in any dimension.

\section{CONCLUSION}

In the present paper, we have studied a model for pulsed laser deposition with and without Ehrlich-Schwoebel barri- ers. The model assumes that the particles are deposited at thermal energies, where the transient mobility of adatoms, edge diffusion, as well as implantation effects can be neglected. First, we investigated the case without EhrlichSchwoebel barriers. For low pulse intensities, PLD displays essentially the same behavior as MBE. Increasing the intensity, it crosses over to a different behavior characterized by the nucleation of many small islands after the first pulse. The behavior in this regime can be analyzed by studying the time-dependent nucleation density. Extending previous results, we have shown that in the limit $D / F \rightarrow \infty$, this quantity displays an unusual type of logarithmic scaling behavior [32].

Turning to PLD with Ehrlich-Schwoebel barriers and without leakages, we first showed that in the limit $D / F$ $\rightarrow \infty$, the second layer nucleation probability grows with increasing intensity, enhancing the Villain instability. This means that in this limit, PLD cannot improve layer-by-layer growth. For finite $D / F$, however, the situation is different. Studying the surface width for the Schwoebel lengths between 1 and $10^{4}$, we found that PLD produces a smoother surface than MBE if the barrier is strong enough. This reversal can be explained by the influence of the initial nucleation density after the first pulse on the roughening due to the Villain instability. However, we believe that this mechanism alone cannot explain the experimentally observed improvement of layerwise growth in PLD, where the barriers are much lower. For example, in the experiments of Ref. [6], where $\mathrm{Fe}$ was deposited on $\mathrm{Cu}(111)$, the additional energy barrier for hopping across edges amounts to less than $20 \%$ of the diffusion barrier, i.e., the Schwoebel length is of the order of a lattice constant. Thus, the observed improvement of the deposited film has to rely on a different mechanism in this case.

As we pointed out, Schinzer et al. [24] had reached a similar conclusion that pulsed deposition leads to increased roughness. However, this statement, which contradicts an idea by Rosenfeld et al. [25] cannot be universally valid, as our more detailed investigation has shown. In our opinion, the negative effect of pulses is mainly due to the fact that the probability of multiple deposition on top of islands is increased. This indicates, that the very high intensity of the single pulse per monolayer was responsible for the roughness in the simulations of Schinzer et al. [24].

However, Schinzer et al. [24] also discovered a way to improve MBE with pulsed deposition: If desorption cannot be neglected, atoms will preferentially evaporate from the top terraces. Thereby, the negative effect of multiple deposition can be compensated, so that one gains the benefit of enforcing small islands by the pulse in the beginning of the monolayer.

Our model describes only a limiting case of pulsed laser deposition. It has the virtue of making new scaling concepts clear. In practice, the atoms are seldomly deposited with thermal energy in pulsed laser deposition. In particular, it would be interesting to work out the effect of transient mobility due to heating by the pulse. Such an extension would allow us to access the range of moderate energies between 1 


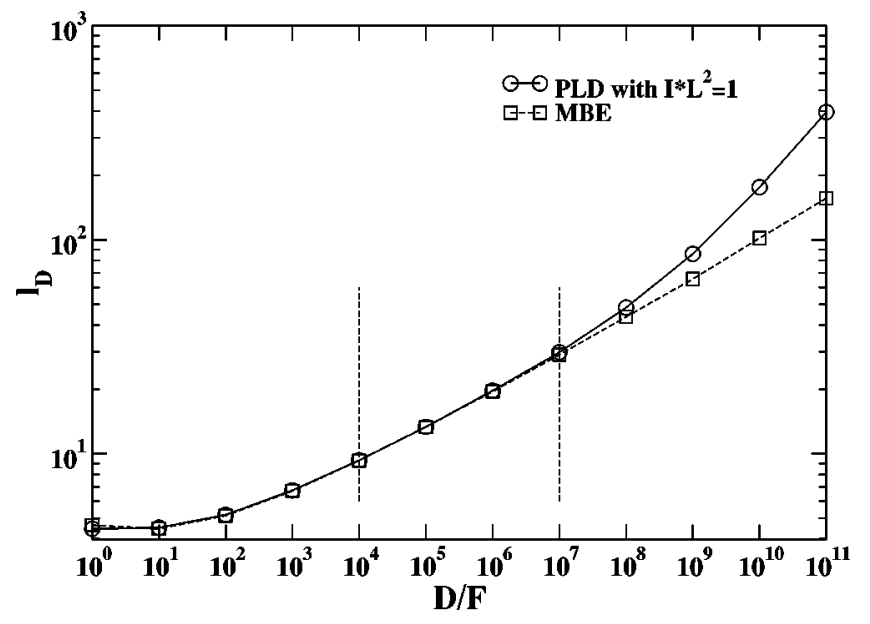

FIG. 10. Island distance for MBE and PLD with $I L^{2}=1$ as a function of $D / F$ for a $400 \times 400$ lattice. The island distance is measured at $0.2 \mathrm{ML}$.

and $10 \mathrm{eV}$, which was used in the experiments by Jenniches et al. [6].

\section{ACKNOWLEDGMENTS}

We would like to thank J. Krug for pointing out Refs. $[13,14]$. We also thank the Deutsche Forschungsgemeinschaft for support under Grant No. SFB 491.

\section{APPENDIX: FINITE-SIZE EFFECTS IN THE LIMIT OF LOW INTENSITY}

As discussed in Sec. II, for small intensities, PLD displays essentially the same growth behavior as MBE. However, on finite lattices, both processes are not exactly equivalent. This can be seen in Fig. 10, where the average island distances for MBE and for PLD with an intensity of one particle per pulse $I=L^{-2}$ are compared over a wide range of $D / F$. For very low values of $D / F$, the curves coincide but do not follow a power law because of lattice effects. Only in the range $10^{4}$ $\leqslant D / F \leqslant 10^{7}$, the curves follow approximately the expected power law $\ell_{D} \propto(D / F)^{\gamma}$. Finally, for $D / F \geqslant 10^{7}$, the island distances of MBE and PLD differ increasingly from each other.

In order to demonstrate that this discrepancy between PLD and MBE is a finite-size effect, we determine the values of $(D / F)_{\mathrm{dev}}$, from where on the island distances in both models differ by a certain factor. As shown in Fig. 11, these values increase algebraically with the system size as

$$
(D / F)_{\operatorname{dev}} \propto L^{\chi},
$$

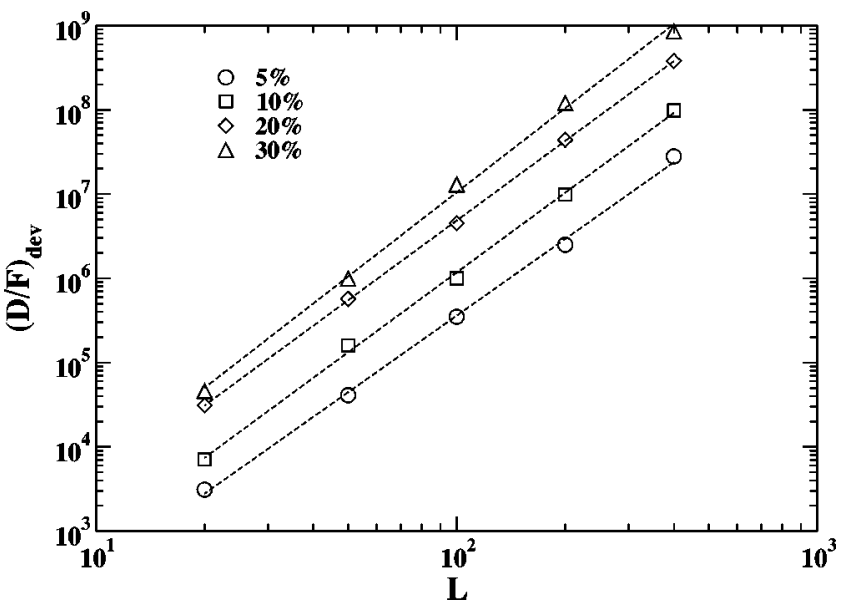

FIG. 11. Values of $D / F$ where the island distances in PLD with $I L^{2}=1$ and in MBE differ at least by a certain percentage specified in the legend. The slopes of the power-law fits from bottom to top are estimated by $\chi=3.0(2), 3.1(2), 3.1(2)$, and 3.2(2).

where the exponent $\chi$ is found to be close to 3 . This powerlaw behavior can be explained as follows. In both cases, single atoms are deposited and the average time between two depositions $\tau=1 / L^{2}$ is the same. However, in PLD, the deposition takes place in constant time intervals, whereas in MBE, atoms are randomly deposited so that the time intervals between deposition events obey a Poissonian distribution $P(\tau)=L^{2} e^{-L^{2} \tau}$, with the variance $\sigma=\sqrt{\left\langle\tau^{2}\right\rangle-\langle\tau\rangle^{2}}$ $=1 / L^{2}$. If such a fluctuation leads to a $\tau$ which is smaller than the average time interval between two depositions, more nucleations will be produced. As the formation of nucleations is irreversible, this enhancement is not compensated by fluctuations in opposite direction where $\tau$ is large. Therefore, the influence of fluctuations increases the number of nucleations, leading to a smaller average island distance than in PLD. However, this effect can only be seen if the fluctuations are strong enough, i.e., they have to be at least of the same order as $\ell_{D}^{2} /(D / F)$, which is the average diffusion time (in ML) before an adatom reaches the edge of an island or another adatom. Since $\ell_{D} \sim(D / F)^{\gamma}$, this argument leads to the scaling relation

$$
(D / F)_{\operatorname{dev}} \propto L^{\chi}, \quad \chi=\frac{2}{1-2 \gamma} .
$$

For $\gamma=1 / 6$, the exponent is $\chi=3$ while for diffusion-limited aggregation, it is given by $\chi \simeq 3.1$. This result is in fair agreement with the numerically determined exponents in Fig. 11.
[1] D.B. Chrisey and G.K. Hubler, Pulsed Laser Deposition of Thin Films (Wiley, New York, 1994).

[2] F.L. Xiong, Y.Y. Wang, V. Leppert, and R.P.H. Chang, J. Mater. Res. 8, 2265 (1993).

[3] H. Tabata, H. Tanaka, and T. Kawai, Appl. Phys. Lett. 65, 1970
(1994).

[4] T.J. Jackson and S.B. Palmer, J. Phys. D 27, 1581 (1994).

[5] D.H. Lowndes, D.B. Geohegan, A.A. Puretzky, D.P. Norton, and C.M. Roleau, Science 273, 898 (1996).

[6] H. Jenniches, M. Klaua, H. Höche, and J. Kirschner, Appl. 
Phys. Lett. 69, 3339 (1996).

[7] R.D. Narhe, M.D. Khandkar, K.P. Adhi, A.V. Limaye, S.R. Sainkar, and S.B. Ogale, Phys. Rev. Lett. 86, 1570 (2001).

[8] P. Jensen and B. Niemeyer, Surf. Sci. 384, L823 (1997); N. Combe and P. Jensen, Phys. Rev. B 57, 15553 (1998).

[9] F. Westerhoff, L. Brendel, and D.E. Wolf, in Structure and Dynamics of Heterogeneous Systems, edited by P. Entel and D.E. Wolf (World Scientific, Singapore, 2000); B. Hinnemann, F. Westerhoff, and D.E. Wolf, Phase Transit. 75, 151 (2002).

[10] B. Hinnemann, H. Hinrichsen, and D.E. Wolf, Phys. Rev. Lett. 87, 135701 (2001).

[11] J. Villain, A. Pimpinelli, and D.E. Wolf, Comments Condens. Matter Phys. 16, 1 (1992); A. Pimpinelli and J. Villain, Physics of Crystal Growth (Cambridge University Press, Cambridge, London, 1998).

[12] P. Meakin, in Phase Transitions and Critical Phenomena, edited by C. Domb and J. Lebowitz (Academic Press, London, 1988), Vol. 12, p. 335.

[13] L.P. Kadanoff, S.R. Nagel, L. Wu, and S.-M. Zhou, Phys. Rev. A 39, 6524 (1988).

[14] C. Tang, NSF-ITP-89-118 (unpublished).

[15] S.B. Lee (private communication).

[16] P. Krapivsky (private communication).

[17] L.H. Tang, J. Phys. I 3, 935 (1993).

[18] G. Ehrlich and F.G. Hudda, J. Chem. Phys. 44, 1039 (1966); R.L. Schwoebel and E.J. Shipsey, J. Appl. Phys. 37, 3682 (1966); R.L. Schwoebel, ibid. 40, 614 (1969).

[19] P. Śmilauer and S. Harris, Phys. Rev. B 51, 14798 (1995).

[20] J. Krug, Adv. Phys. 46, 139 (1997).
[21] J. Villain, J. Phys. I 1, 19 (1991).

[22] M. Siegert and M. Plischke, Phys. Rev. Lett. 68, 2035 (1992); 73, 1517 (1994).

[23] J.A. Stroscio, D.T. Pierce, and R.A. Dragoset, Phys. Rev. Lett. 70, 3615 (1993); J.A. Stroscio and D.T. Pierce, Phys. Rev. B 49, 8522 (1994); J.E. van Nostrand, S.J. Chey, M.A. Hasan, D.G. Cahill, and J.E. Greene, Phys. Rev. Lett. 74, 1127 (1995); H.-J. Ernst, F. Fabre, R. Folkerts, and J. Lapujoulade, ibid. 72, 112 (1994); K. Thurmer, R. Koch, M. Weber, and K.H. Rieder, ibid. 75, 1767 (1995).

[24] S. Schinzer, M. Sokolowski, M. Biehl, and W. Kinzel, Phys. Rev. B 60, 2893 (1999).

[25] G. Rosenfeld, N.N. Lipkin, W. Wulfhekel, J. Kliewer, K. Morgenstern, B. Poelsema, G. Comsa, Appl. Phys. A: Mater. Sci. Process. 61, 455 (1995).

[26] J. Tersoff, A.W. Denier van der Gon, and R.M. Tromp, Phys. Rev. Lett. 72, 266 (1994).

[27] J. Krug, P. Politi, and T. Michely, Phys. Rev. B 61, 14037 (2000).

[28] D.E. Wolf, in Scale Invariance, Interfaces and NonEquilibrium Dynamics, edited by A. McKane et al. (Plenum Press, New York, 1995).

[29] H. Kallabis, L. Brendel, J. Krug, and D.E. Wolf, Int. J. Mod. Phys. B 11, 3621 (1997).

[30] L. Brendel, Ph.D. thesis, Gerhard-Mercator Universität Duisburg, 2001 (unpublished).

[31] E. Somfai, D.E. Wolf, and J. Kertész, J. Phys. I 6, 393 (1996).

[32] L. Sittler and H. Hinrichsen, J. Phys. A 35, 10531 (2002). 